\title{
RESEARCH ON METHANE LEAK DETECTION TECHNOLOGY BASED ON INFRARED ABSORPTION PRINCIPLE
}

\author{
X.Y.Chen
}

College of Electrical and Information

Southwest Petroleum University, Chengdu, Sichuan, China

$$
\text { Y.Peng }
$$

College of Electrical and Information

Southwest Petroleum University, Chengdu, Sichuan, China

Q.Miao

College of Electrical and Mechanical Engineering Southwest Petroleum University, Chengdu, Sichuan, China
L.Ge

College of Electrical and Mechanical Engineering Southwest Petroleum University, Chengdu, China

X.Y.Zheng

College of Electrical and Information Southwest Petroleum University, Chengdu, China

Y.J.Chen

College of Electrical and Information Southwest Petroleum University, Chengdu, China,

\author{
L.Huang \\ College of Electrical and Information \\ Southwest Petroleum University, Chengdu, Sichuan, China
}

\begin{abstract}
As a clean energy, natural gas accounts for an increasing proportion of my country's energy consumption structure. Due to the flammable and explosive characteristics of natural gas, natural gas leakage can easily cause safety accidents. This paper has designed a gas concentration detection system based on the principle of infrared absorption, which can detect the methane concentration in natural gas, quickly determine whether there is a methane leak, and effectively protect people's lives and property.
\end{abstract}

Keywords - Infrared Absorption; Methane Gas; Leak Detection

\section{INTRODUCTION}

With the continuous development of national science and industrial production technology, the energy demand of China is increasing. Natural gas is a kind of clean energy and has abundant reserves, and it accounts for an increasing proportion of energy consumption structure of China. Because natural gas is inflammable and explosive, natural gas leakage can easily cause safety accidents and huge economic losses and may even threaten people's lives [1]. In addition, the methane in natural gas is a greenhouse gas, and natural gas leakage will gradually increase the concentration of methane in the air, which is also a factor that causes the greenhouse effect to be more serious [2]. The leakage of natural gas will not only bring safety problems, but also environmental protection problems, therefore the detection of methane gas leakage is very necessary [3].

The methane leakage detection system designed in this paper is based on the principle of infrared absorption, which meets the needs of current national development. It has the characteristics of low cost, good stability, and high precision, and has broad application prospects.

\section{THEORY OF THE SYSTE}

Differential absorption detection method is one of the commonly used gas concentration detection methods, which is usually divided into single-wavelength dual-path method and dual-wavelength single-path method [4]. As shown in Fig. 1, in the dual-wavelength single-path method, the infrared light passes through the gas chamber filled with the gas to be measured, and then the infrared light is received by the sensor equipped with two filters. Since the two filters of the sensor only allow infrared light with wavelengths of and to pass through, the channels with wavelengths of and are used as the measurement channel and the reference channel. The two signals are input into two signal processing circuits respectively, and then the gas concentration is calculated by making a ratio in the single-chip microcomputer. In theory, the ratio information of the two channels can eliminate the influence of external factors, thereby improving the accuracy of gas concentration detection [5]. 


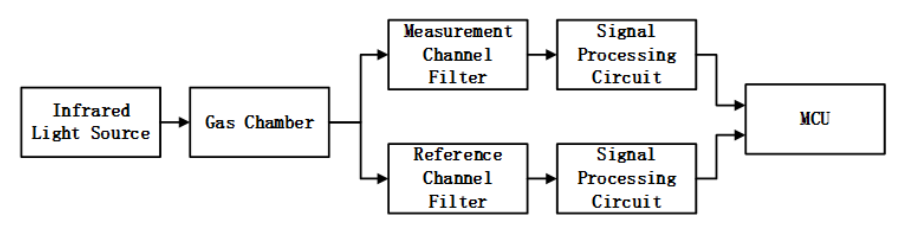

Fig. 1. Principle block diagram of dual-wavelength single-path method

\section{HARDWARE AND SOFTWARE DESIGNS OF THE SYSTE}

\section{A. Hardware software of the system -}

As shown in Fig. 2, the hardware part of methane leakage detection device can be divided into two parts: optical measurement part and circuit processing part. The optical measurement part includes light source modulation circuit, infrared light source, gas chamber and pyroelectric infrared sensor. The circuit processing part includes filter and amplifier circuit, A/D conversion circuit, micro-control processor and LCD display circuit.

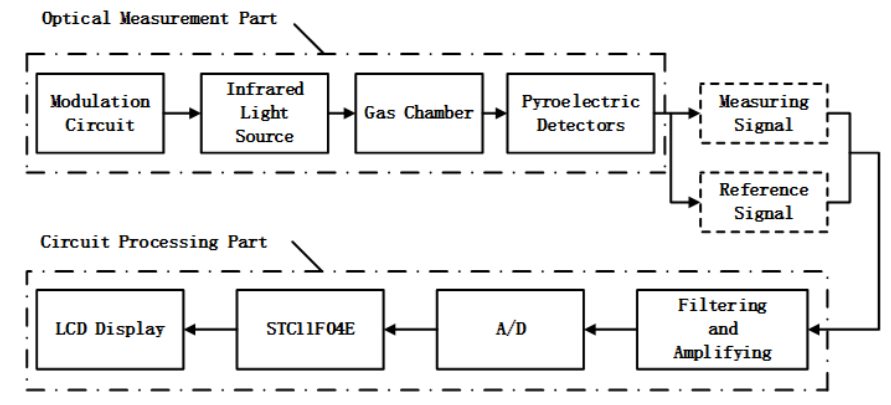

Fig. 2. Hardware block diagram of the methane leak detection device

The processor of the system only needs to achieve some simple control functions, and the use of large-scale programmable logic devices will increase unnecessary operations and high cost, so the MCU STC11F04E is chosen as the control processor of the system. STC11F04E is a single mechanical cycle MCU produced by STC, which integrates large-capacity RAM and ROM, with the characteristics of high speed, strong anti-interference, low cost and ultra-low power consumption. It is a kind of enhanced 8051 single-chip microcomputers, and the execution speed of instruction code is many times faster than 8051 , therefore it is commonly used in intelligent control and high-speed communication [6].

Methane gas has an absorption peak at $3.31 \mu \mathrm{m}$, while other components in the air have no absorption peak at $3.31 \mu \mathrm{m}$, so the wavelength range of infrared light source should include $3.31 \mu \mathrm{m}$, which can greatly reduce the interference of other components in the air to the measurement results and improve the accuracy of measurement [7]. In this system, the IRL715 thermal radiation infrared light source is selected as the signal source with small size and long service life, whose wavelength range is from visible light to $4.4 \mu \mathrm{m}$ and modulation frequency can reach up to $3 \mathrm{~Hz}[8]$.

The two channels of the pyroelectric infrared sensor PYS3228 G5.2/G20 can output voltage signals, and each channel has a separate filter window, whose central wavelength of the filter window is $3.3 \mu \mathrm{m}$ or $3.95 \mu \mathrm{m}$. The horizontal field of view of the sensor can reach $77^{\circ}$, and it is durable, fast in response, low in noise, and low in cost [9]. Since infrared light sensor is not sensitive to stable light intensity, but is sensitive to changes in light intensity, it is usually necessary to modulate the light source when using pyroelectric infrared sensor.

The optical signal received by the infrared sensor PYS3228 is relatively weak, so the output electrical signal is also relatively weak and mixed with noise signals [10]. It is necessary to filter and amplify the original signals of the two channels. The filtering accuracy of the active filter circuit is higher than that of the passive filter circuit, and the active filter circuit has a certain voltage gain and a good filtering effect, which is generally used in the occasions with higher requirements, so the system uses an active filter circuit to filter the original signal. The filter circuit of the measurement channel is shown in Fig. 3. In addition, because the output signals of the sensor contain a fixed DC bias voltage [11], it is necessary to eliminate the interference generated by the DC bias voltage before A/D acquisition. Therefore, we use a differential amplifier circuit to amplify the filtered signal. The Amplifier circuit of the measurement channel is shown in Fig. 4.

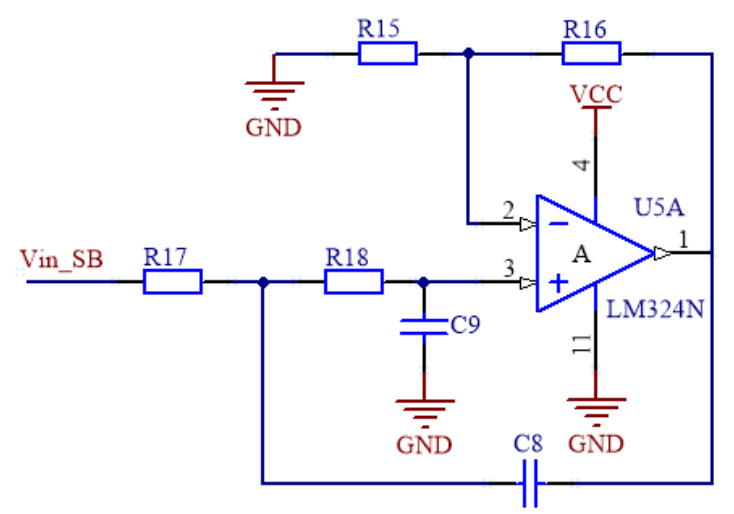

Fig. 3. Filter circuit diagram of the measurement channel 


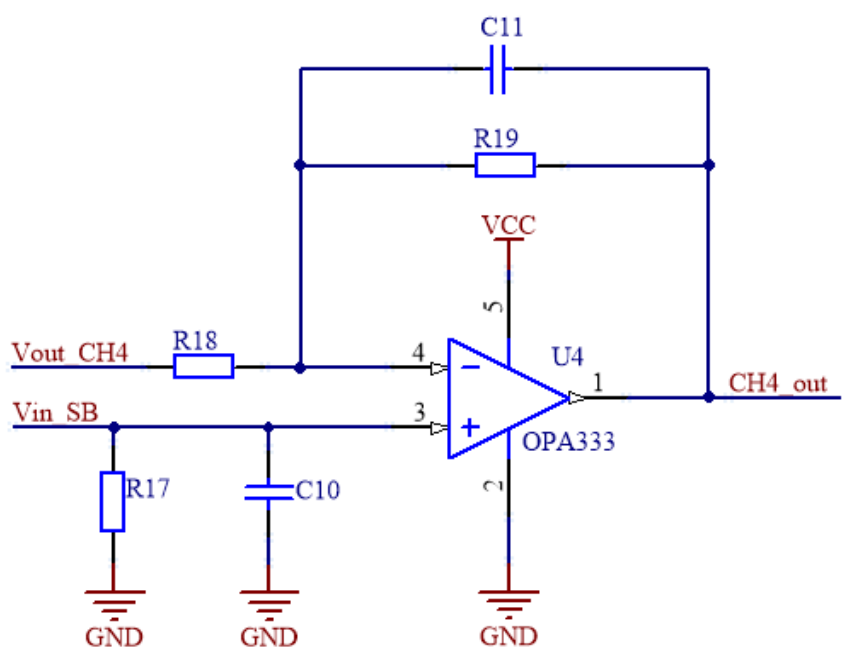

Fig. 4. Amplifier circuit of the measurement channel

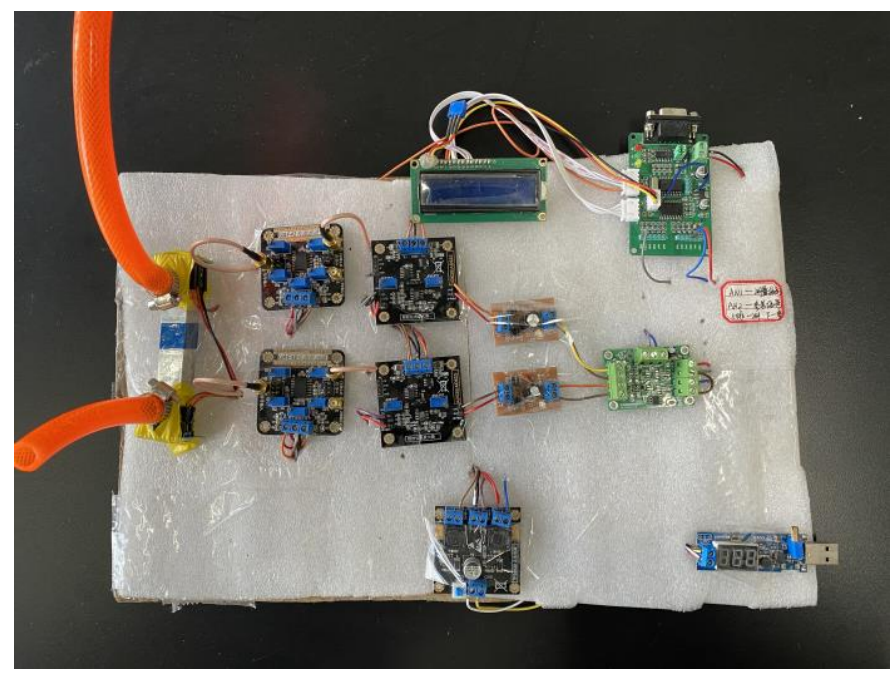

Fig. 5. The physical picture of the detection device

\section{$B$. Software software of the system -}

The main program contains several subroutines. After entering the main program, the MCU first initializes the settings and starts the timer, then the MCU modulates the infrared light source to emit infrared light at $1 \mathrm{~Hz}$ frequency. After collecting the data of the two channels respectively for 20 times and calculating the average value, the MCU processes the converted voltage data to obtain the methane concentration value, and then displays it on the LCD screen. The main program flow chart is shown in Fig. 6.

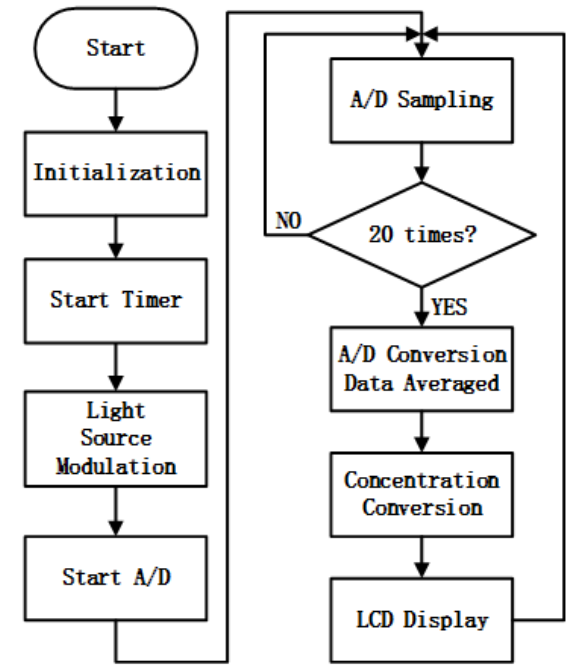

Fig. 6. Main program flow chart

\section{Software software of the system -}

\section{CONCLUSION}

The methane leakage detection system designed in this paper based on the principle of infrared absorption has the characteristics of low cost, simple structure, easy operation, and stability. It can accurately measure whether there is a natural gas leak in the house or industry, effectively reducing safety hazards, reducing economic losses and ensuring people's life safety, and has strong practical value.

\section{REFERENCE}

[1] Yu Li. Numerical simulation of natural gas leakage, diffusion and explosion in confined space[D]. Capital University of Economics and Business, 2011.

[2] Tang Jianhua. The hidden greenhouse gas-methane[J]. Chemical Education, 1990, 11(6): 4-6.

[3] Wang Haixu. Design of high-precision portable methane detection device [D]. Jilin University, 2017.

[4] Zhang Yu, Wang Yiding, Li Li, et al. Analysis of the principle and processing technology of methane infrared absorption spectroscopy[J]. Spectroscopy and Spectral Analysis, 2008, 28(11): 2515-2519.

[5] Jingsong Li,Uwe Parchatka,Horst Fischer. Development of field-deployable QCL sensor for simultaneous detection of ambient N $2 \mathrm{O}$ and $\mathrm{CO}[\mathrm{J}]$. Sensors \& Actuators: B. Chemical,2013,182.

[6] Shen Jianguang.Research on the detection method of infrared methane gas sensor[J].Metrics and Testing Technology,2018,45(07):90-93. 
[7] Sharpe S W , Johnson T J , Sams R L, et al. Gas-phase databases for quantitative infrared spectroscopy.[J]. Applied Spectroscopy, 2004, 58(12):1452-1461.

[8] Fawcett B L, Parkes A M , Shallcross D E, et al. Trace detection of methane using continuous wave cavity ringdown spectroscopy at $1.65 \mu \mathrm{m}[\mathrm{J}]$. Physical Chemistry Chemical Physics, 2002, 4(24):5960-5965.

[9] Xue Chenyang, Tan Qiulin, Ma Youchun, Qin Haitao, Zhai Chengrui, Zhang Wendong.The design of infrared sensor signal extraction and data acquisition[J].Instrument Technology and Sensors,2007(02):45-46+49.

[10] Fu Hua,Yang Yikui,Liu Yujia.Experimental research on the new technology of double difference method for detecting gas content[J].Journal of China Coal Society,2012,37(07):1161-1164.

[11] Wang Y, Nakayama M , Yagi M, et al. The NDIR CO2 monitor with smart interface for global networking $[\mathrm{J}]$. IEEE Transactions on Instrumentation and Measurement, 2005, 54 (4):1634-1639.

[12] Li S , Li X, Yang J , et al. Novel Reflection-type Optical Fiber Methane Sensor Based on a No-core Fiber Structure[J]. Materials today: proceedings, 2016, 3(2):439-442.

[13] Chen Youan. Design of methane gas online monitoring system based on infrared spectrum absorption[D]. University of Science and Technology of China, 2016. 